\title{
Efficiency evaluation of unattended convenience store based on bi-level DEA with multi-followers
}

\author{
Zhuang Yang ${ }^{* 1, \mathrm{a}}$, Li Hui ${ }^{1}$,Xudong Chen $^{2}$ \\ ${ }^{1}$ Business school, Sichuan University Chengdu, 610065, China \\ ${ }^{2}$ College of Management Science, Chengdu University of Technology Chengdu, 610059, China
}

\begin{abstract}
As an application of digital concepts, unattended convenience stores are widely disseminated and considered one of the trends of future business development. It is an important consideration for the development of unattended convenience stores to determine the potential of operation efficiency and improve inefficient units. This paper establishes a bi-level DEA model with multi-followers and uses weak disposability technology to deal with undesirable intermediate measures, aiming to accurately evaluate the performance of the whole bi-level system of unattended convenience stores and each participant. Thus, extended Kuhn-Tucker conditions are applied to solve the proposed model. It is found that the benefit efficiency of the lower subsystem always belongs to the maximum and minimum benefit efficiency of all followers, and the benefit efficiency of the system is between the upper and lower benefit efficiency. Using a case study of unattended convenience stores in the Chengdu area, the validity and practicability of the model in the evaluation of benefit efficiency are shown and more explicit management opinions are provided.
\end{abstract}

\section{Introduction}

With the application of artificial intelligence technology, the development of third-party payments and the upgrading of consumer markets, there is a trend that machines are replacing manpower in all walks of life. In China, unattended convenience stores are becoming one of the most popular investment areas after bicycle sharing. For example, the bi-level system consisting of a control center and multiple retail boxes distributed around it is the most common management mode of unattended convenience stores. The unattended convenience store is a newly created business entity, and its efficiency evaluation has important guiding significance and commercial value for practice and development. However, as far as we know, there has been no attempt to evaluate the efficiency of unattended convenience stores. When such a bi-level system is evaluated, it is necessary to consider the interaction and structure of the participants in the system. Therefore, an appropriate method for evaluating such a system is required.

At present, data envelopment analysis (DEA) is one of the most widely used mathematical methods for evaluating the relative efficiency of decision making units (DMU) in multi-input and multi-output situations. Charnes, et al. [1] proposed CCR-DEA in 1978 to evaluate the relative efficiency of similar DMUs with multiple inputs and outputs, which has advantages in avoiding subjective factors and simplifying algorithms. Banker, et al. [2] extends the work of Charnes, et al. [1] based on the assumption of variable returns to scale of a possible production set, and proposes a BCC-DEA model. To evaluate the efficiency of the system more accurately, some authors propose dividing the system into several stages. The efficiency of the system can be reflected by measuring the efficiency of each stage in the system. Fare and Whittaker [3] and Fare and Grosskopf [4] inaugurated the two-stage DEA model field and studied the relative efficiency of dairy production using the two-stage DEA model oriented to input. To date, numerous scholars have applied the two-stage model to efficiency evaluation in various situations. Chen, et al. [5] established a DEA model using the additive efficiency decomposition of twostage network processes to measure the performance of two-stage network processes with indivisible shared inputs. Liang, et al. [6] introduced the concept of game theory into two-stage network DEA research. Under the background of a seller-buyer supply chain, Liang, et al. [6] developed a DEA model to evaluate supply chain performance by applying leader-follower and cooperative relationship theory respectively.

In studying the two-stage DEA, the participants in the system are usually regarded as equal and independent, and their performance is measured by applying different DEA models to different participants. However, participants in the bi-level system are at different levels, and they operate according to Nash Stackelberg game rules within the system. The leader first makes a decision to optimize his goals, which will affect his followers' actions, and followers' reactions may partly determine his goals.

A new bi-level programming DEA method was proposed by $\mathrm{Wu}$ [7], which enables us to evaluate the performance of enterprises in decentralized decision-

\footnotetext{
a*Corresponding author: 951662017@qq.com
} 
making and is confirmed by banking chain and manufacturing supply chain case studies. Wu, et al. [8] measured banking merger evaluation by developing a leader-follower game model, demonstrating how incentive-compatible business mergers can improve overall efficiency. It can be concluded that the bi-level DEA model can effectively handle a bi-level system with a leader and a follower, whereas in some bi-level systems, there is not only one player at each level. For example, in an unattended convenience store system, there are several lower-level retail boxes that use system resources but operate independently. For the bi-level multiple followers decision-making problem, $\mathrm{Lu}$, et al. [9] established a general framework, and put forward relevant theories, especially for a non-cooperative decision-making problem. On this basis, Zhou, et al. [10] proposed a new bi-level DEA model with multiple followers, which can evaluate the cost efficiency of the entire bi-level system and each participant. Although their models can solve the problem of efficiency evaluation of general bi-level multi-follower systems, similar to most studies, they only focus on the desirable output of each level, but neglect to deal with the undesirable output that may occur in the system.

This paper extends the bi-level DEA model with multiple followers proposed by Zhou, et al. [10]. By using the principle of weak disposability to deal with the undesirable output generated by the upper subsystem, which is also regarded as input of the lower subsystem, the new model can effectively solve the efficiency evaluation problem of the bi-level multiple followers system with shared resources and undesirable intermediate measures. In addition, in order to reflect the output efficiency of unattended convenience stores more comprehensively and accurately and to provide managers with management insights and best practices, we propose a new efficiency evaluation index, Benefit Efficiency (BE). Unlike profit efficiency, benefit efficiency considers all undesirable outputs of all participants in the system. This study has important guiding significance for the optimization and development of unattended convenience stores.

The rest of the article is organized as follows. Section 2 describes the problem and the framework of modeling the unattended convenience store system. Section 3 presents the preliminaries of benefit efficiency DEA. Section 4 develops the model and sets forth the definitions. Section 5 discusses the solution of the bi-level model. Section 6 uses the model to evaluate the unattended convenience store system in detail. The conclusion section summarizes the findings of the study.

\section{Preliminaries}

This section presents a benefit-efficiency CCR DEA model and the treatment of undesirable measures. Suppose that there are I DMUs, indexed by $j(j=1,2, \ldots, J)$ and each of them has R inputs $x_{r j},(r=1,2, \ldots, R)$ for producing I $y_{r j},(r=1,2, \ldots, R) \quad$ desirable outputs and $\mathrm{M}$ $y_{m j},(\mathrm{~m}=1,2, \ldots, \mathrm{M})$ undesirable outputs. $w_{i}$ are user- specified weights such that $\sum_{i=1}^{I} w_{i}=1$. To measure the performance of DMU0 with multiple inputs and outputs, we use the following linear programming to maximize desirable output with current input, simultaneously controlling the undesirable output.

$$
\begin{array}{r}
W Y^{*}=\max \sum_{i=1}^{m} w_{i} y_{i} \\
\text { s.t. }\left\{\begin{array}{l}
x_{r 0} \geq \sum_{j=1}^{n} x_{r j} \alpha_{j}, r=1, \ldots, R \\
y_{i} \leq \sum_{j=1}^{n} y_{i j} \alpha_{j}, i=1, \ldots, I \\
y_{m}=\sum_{j=1}^{n} y_{m j} \alpha_{j}, m=1, \ldots, M \\
\alpha_{j} \geq 0, j=1, \ldots, J
\end{array}\right.
\end{array}
$$

where $\sum_{i=1}^{m} w_{i} y_{i}$ is the maximum desirable output of DMU that can be obtained at the current input, while keeping the level of the undesirable output unchanged, subject to the resources constraints imposed by the existing production-possibility frontier. The restriction $y_{m}=\sum_{j=1}^{m} y_{m j} \alpha_{j} \quad$ means to control the undesirable output, which is preferable to the "weak disposability" concept.

Based on the optimal solution $\left(y^{*}, \alpha^{*}\right)$ of the model (1), the benefit efficiency (BE) of the evaluated DMU0 is defined as the ratio of current benefit and the optimal benefit as follows:

$$
B E=\frac{W Y_{0}}{W Y^{*}}
$$

where $\mathrm{BE}$ satisfies $0<B E \leq 1$. If $B E=1$, then DMU0 is efficient; otherwise, it is inefficient.

\section{Modeling}

In this section, we develop a multiple-followers BLP-DEA model with undesirable intermediate measures to evaluate the performance of an unattended convenience store system and its participants. The detailed structure of the system is shown in Figure 1. Based on the evaluated BE of the lower-level model of DMUs, the upper-level model is concerned with the coordination and allocation of resources to maximize the benefits of the entire system. This model considers the treatment of shared input and undesirable intermediate measures.

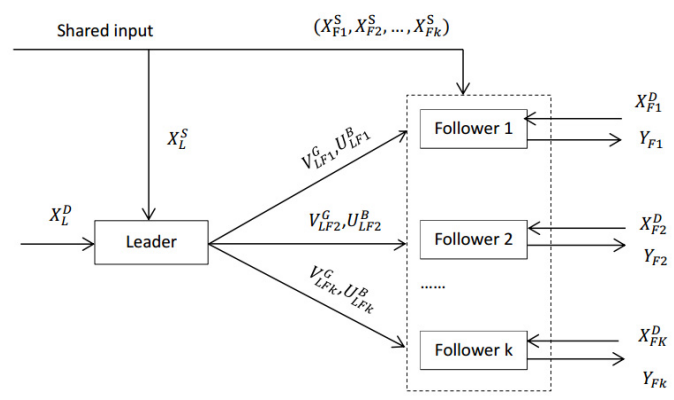

Figure 1. Structure of a bi-level system 


\subsection{Notation}

Before establishing the model, we define some notations as follows:

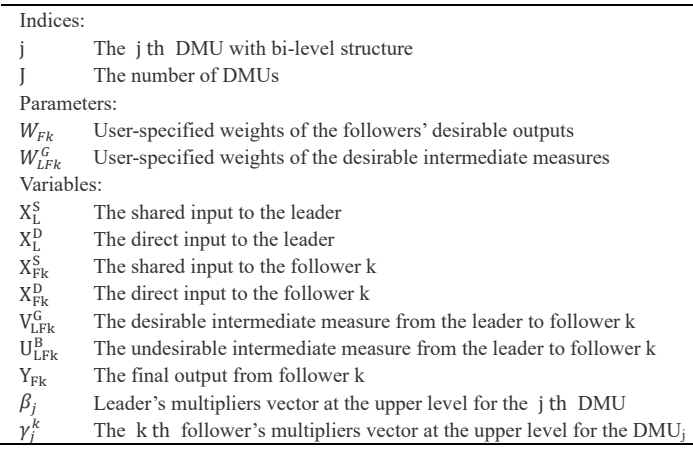

\subsection{Multiple followers BLP-DEA model with undesirable intermediate measures}

In the bi-level programming model, in order to achieve its goal, the optimal solution obtained by lower-level programming must be considered one of the upper-level constraints.

Thus, the multiple followers BLP-DEAwUIM are presented as:

$$
\begin{aligned}
& \frac{\max }{V_{L F k}^{G}, Y_{F k}, \beta}=\sum_{k=1}^{K} W_{L F k}^{G} \overline{V_{L F k}^{G}}+\sum_{k=1}^{K} W 1_{F k} \overline{Y 1_{F k}}+\sum_{k=1}^{K} W 2_{F k} \overline{Y 2_{F k}}
\end{aligned}
$$

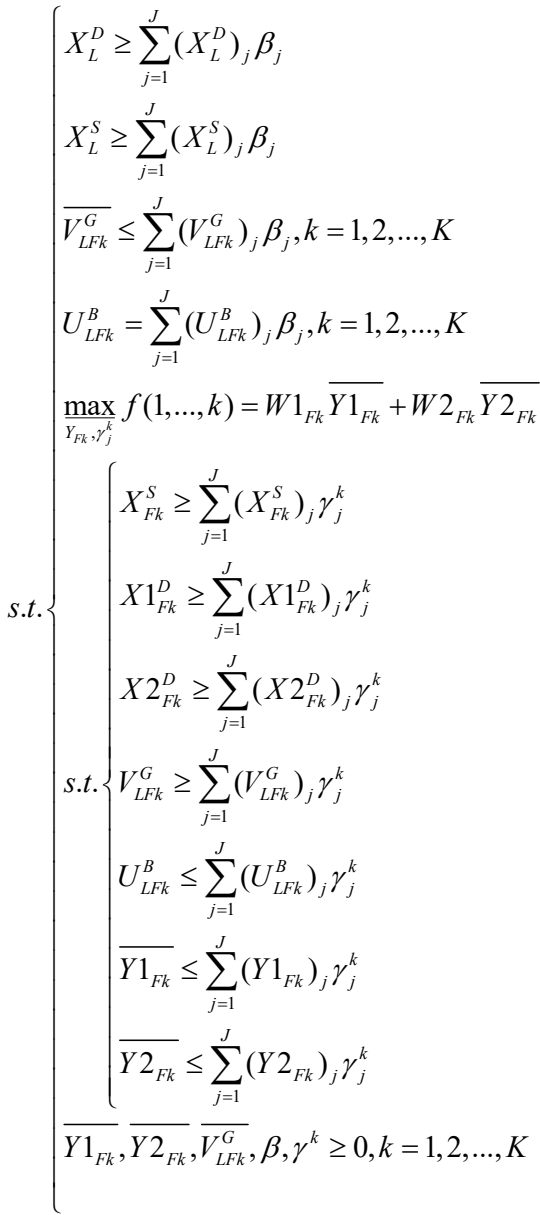

Note that in the above formula, both the intermediate measures $U_{L F k}^{B}$ and $V_{L F k}^{G}$ play two roles at the same time: not only the output of the leader but also the input of followers.

The vehicle routing problem with capacity constraints can be described as: there is a distribution center with $\mathrm{M}$ vehicles of the same model, and the maximum load of each vehicle is $Q$. these vehicles start from the distribution center to serve $\mathrm{K}$ demand points, the demand of demand point $\mathrm{i}$ is $\mathrm{d}_{\mathrm{i}}$. Each demand point can only be visited by one vehicle, and each vehicle must return to the distribution center after service. The objective function is to find a vehicle distribution scheme that satisfies the constraint conditions, which can meet the needs of customers and minimize the total transportation distance and total transportation cost.

\subsection{Definitions}

The following benefit efficiency definitions are shown to assess the performance of J DMUs with a bi-level structure described in this article.

Definition 1. (Benefit efficiency of the upper level) The BE of the upper level subsystem, also described as the leader's BE for $D M U_{j}$, is defined as

$$
B E_{j}^{U}=\frac{\sum_{k=1}^{K} W_{L F k}^{G}\left(V_{L F k}^{G}\right)_{j}}{\sum_{k=1}^{K} W_{L F k}^{G} \overline{\left(V_{L F k}^{G}\right)_{j}} *}
$$

where the $\overline{\left(V_{L F k}^{G}\right)_{j}}$ * is the optimal solution by solving the model (2) for $D M U_{j}$.

Definition 2. (Benefit efficiency of the lower level) The $\mathrm{BE}$ of the lower level subsystem for $D M U_{j}$ is defined as

$$
B E_{j}^{L}=\frac{\sum_{k=1}^{K} W 1_{F k}\left(Y 1_{F k}\right)_{j}+\sum_{k=1}^{K} W 2_{F k}\left(Y 2_{F k}\right)_{j}}{\sum_{k=1}^{K} W 1_{F k}\left(\overline{Y 1_{F k}}\right)_{j} *+\sum_{k=1}^{K} W 2_{F k}\left(\overline{Y 2_{F k}}\right)_{j} *}
$$

where the $\left(\overline{Y 1_{F k}}\right)_{j} *$ and $\left(\overline{Y 2_{F k}}\right)_{j} * \quad$ is the optimal solution by solving the model (2) for $D M U_{j}$.

Definition 3. (Benefit efficiency of the $D M U_{j}$ 's $k$ th follower) The $\mathrm{BE}$ of $D M U_{j}$ 's follower $\mathrm{k}$ is defined as

$$
B E_{j}^{F k}=\frac{W 1_{F k} Y 1_{F k}+W 2_{F k} Y 2_{F k}}{W 1_{F k}\left(\overline{Y 1_{F k}}\right)_{j} *+W 2_{F k}\left(\overline{Y 2_{F k}}\right)_{j}^{*}}
$$

Definition 4. (Benefit efficiency of the entire system) The overall $\mathrm{BE}$ of the unattended convenience store for $D M U_{j} \quad$ is defined as

$$
B E_{j}=\frac{\sum_{k=1}^{K} W_{L F k}^{G}\left(V_{L F k}^{G}\right)_{j}+\sum_{k=1}^{K} W 1_{F k}\left(Y 1_{F k}\right)_{j}+\sum_{k=1}^{K} W 2_{F k}\left(Y 2_{F k}\right)_{j}}{\sum_{k=1}^{K} W_{L F k}^{G} \overline{\left(V_{L F k}^{G}\right)_{j}} *+\sum_{k=1}^{K} W 1_{F k}\left(\overline{Y 1_{F k}}\right)_{j} *+\sum_{k=1}^{K} W 2_{F k}\left(\overline{Y 2_{F k}}\right)_{j} *}
$$

\section{Solution method}

The proposed bi-level programming (BLP) model in this paper is an NP-hard nonconvex global optimization problem, which is difficult to solve effectively [11]. To make it solvable, it must be transformed into a single-level 
programming (SLP) problem that can be solved using standard optimization software.

To begin with, it is necessary to transform model (2) into a standard formulation as follows:

$$
\begin{aligned}
& \min F\left(x, y_{1 K}, \ldots, y_{k}\right)=\delta^{U} x+\sum_{k=1}^{K} \theta_{K}^{L} y_{k} \\
& \text { s.t. }\left\{\begin{array}{l}
A^{U} x+\sum_{k=1}^{K} B_{k}^{U} y_{K} \leq e_{1}^{U} \\
C x+\sum_{k=1}^{K} D_{k}^{U} y_{k}=e_{2}^{U} \\
\min f_{k}\left(y_{1}, \ldots, y_{k}\right)=\theta_{k}^{L} y_{k}(3) \\
\text { s.t. }\left\{\begin{array}{l}
A_{k}^{L} x_{k}+B_{k}^{L} y_{k} \leq e_{1 k}^{L} \\
C_{k}^{L} x+D_{k}^{L} y_{k}=e_{2 k}^{L}
\end{array}\right. \\
x_{k} \geq 0, y_{k} \geq 0 \\
k=1,2, \ldots K
\end{array}\right.
\end{aligned}
$$

Note that there is k programming at the lower level of the standard form BLP model (3). Where variables and coefficient matrixes are defined as follows:

$$
\begin{aligned}
& x=\left(\begin{array}{l}
\overline{V_{L F k}^{G}} \\
\beta_{j}
\end{array}\right)_{12 \times 1}, y_{k}=\left(\begin{array}{l}
\overline{Y 1_{F k}} \\
\overline{Y 2_{F k}} \\
\gamma_{j}^{k}
\end{array}\right)_{9 k \times 1}, A_{k}^{L}=(0), B_{k}^{U}=(0)
\end{aligned}
$$

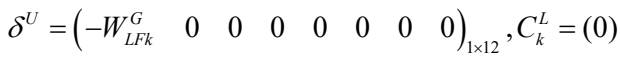

$$
\begin{aligned}
& \theta_{k}^{L}=\left(\begin{array}{lllllllll}
-W 1_{L F k} & -W 2_{L F k} & 0 & 0 & 0 & 0 & 0 & 0 & 0
\end{array}\right)_{1 \times 9 k} \\
& A^{U}=\left(\begin{array}{cccccccc}
0 & 0 & 0 & 0 & 0 & \left(X_{L}^{D}\right)_{1} & \ldots & \left(X_{L}^{D}\right)_{j} \\
0 & 0 & 0 & 0 & 0 & \left(X_{L}^{S}\right)_{1} & \ldots & \left(X_{L}^{S}\right)_{j} \\
1 & 0 & 0 & 0 & 0 & -\left(V_{L F 1}^{G}\right)_{1} & \ldots & -\left(V_{L F}^{G}\right)_{j} \\
0 & 1 & 0 & 0 & 0 & -\left(V_{L F 2}^{G}\right)_{1} & \ldots & -\left(V_{L F 2}^{G}\right)_{j} \\
0 & 0 & 1 & 0 & 0 & -\left(V_{L F 3}^{G}\right)_{1} & \ldots & -\left(V_{L F 3}^{G}\right)_{j} \\
0 & 0 & 0 & 1 & 0 & -\left(V_{L F 4}^{G}\right)_{1} & \ldots & -\left(V_{L F 4}^{G}\right)_{j} \\
0 & 0 & 0 & 0 & 1 & -\left(V_{L F 5}^{G}\right)_{1} & \ldots & -\left(V_{L F 5}^{G}\right)_{j}
\end{array}\right)_{7 \times 12} \\
& e_{1}^{U}=\left(\begin{array}{l}
X_{L}^{D} \\
X_{L}^{S} \\
0 \\
0 \\
0 \\
0 \\
0
\end{array}\right)_{7 \times 1} e_{1 k}^{L}=\left(\begin{array}{l}
X_{F k}^{S} \\
X 1_{F k}^{D} \\
X 2_{F k}^{D} \\
V_{L F k}^{G} \\
0 \\
0
\end{array}\right)_{6 k \times 1} \quad e_{2}^{U}=\left(U_{L F k}^{B}\right)_{5 \times 1}, D_{k}^{U}=(0) \\
& C^{U}=\left(\begin{array}{llllllll}
0 & 0 & 0 & 0 & 0 & \left(U_{L F k}^{B}\right)_{1} & \ldots & \left(U_{L F k}^{B}\right)_{7}
\end{array}\right)_{5 \times 12} \\
& B_{k}^{L}=\left(\begin{array}{ccccc}
0 & 0 & \left(X_{F k}^{S}\right)_{1} & \ldots & \left(X_{F k}^{S}\right)_{1} \\
0 & 0 & \left(X 1_{F k}^{D}\right)_{1} & \ldots & \left(X 1_{F k}^{D}\right)_{1} \\
0 & 0 & \left(X 2_{F k}^{D}\right)_{1} & \ldots & \left(X 2_{F k}^{D}\right)_{1} \\
0 & 0 & \left(V_{L F k}^{G}\right)_{1} & \ldots & \left(V_{L F k}^{G}\right)_{1} \\
1 & 0 & -\left(Y 1_{F k}\right)_{1} & \ldots & -\left(Y 1_{F k}\right)_{1} \\
0 & 1 & -\left(Y 2_{F k}\right)_{1} & \ldots & -\left(Y 2_{F k}\right)_{1}
\end{array}\right)_{6 k \times 9 k} \\
& e_{2 k}^{U}=\left(U_{L F k}^{B}\right)_{1 k}, D_{k}^{L}=\left(\begin{array}{lllll}
0 & 0 & \left(U_{L F k}^{B}\right)_{1} & \ldots & \left(U_{L F k}^{B}\right)_{7}
\end{array}\right)_{1 k \times 9}
\end{aligned}
$$

The existing methods to deal with bi-level programming are mainly to replace the lower level problems with the corresponding Karush-Kuhn-Tucker (KKT) conditions [12]. In this paper, we consider using extended Kuhn-Tucker conditions proposed by Shi, et al. [13] to transform model (3) into single-level programming based on Theorem 1, and then use standard optimization software to solve it.

Theorem 1. Denote $p_{k} \in R^{U}, q_{k} \in R^{U}, r_{k} \in R^{U}$ and $t_{k} \in R^{m_{k}},(k=1,2, \ldots, K)$, the row vector dual variables associated with constraints at the upper and the lower level respectively. A necessary and sufficient condition for solving the proposed model (3) with $\left(x_{1}^{*}, \ldots, x_{K}^{*}, y_{1}^{*}, \ldots y_{K}^{*}\right)$ is that there exist row vector $p_{k}^{*}, q_{k}^{*}, r_{k}^{*}$ and $t_{k}^{*}, k=1,2, \ldots, K \quad$, such that $\left(x_{1}^{*}, \ldots, x_{K}^{*}, y_{1}^{*}, \ldots y_{K}^{*}, p_{1}^{*}, \ldots, p_{K}^{*}, q_{1}^{*}, \ldots, q_{K}^{*}, r_{1}^{*}, \ldots, r_{K}^{*}, t_{1}^{*}, \ldots, t_{K}^{*}\right)$ , which can solve:

$$
\begin{aligned}
& \min F\left(x, y_{1}, \ldots y_{k}\right)=\delta^{U} x+\sum_{k=1}^{K} \theta_{k}^{L} y_{k} \\
& \text { s.t. }\left\{\begin{array}{l}
A^{U} x+\sum_{k=1}^{K} B_{k}^{U} y_{k} \leq e_{1}^{U} \\
C^{U} x+\sum_{k=1}^{K} D_{k}^{U} y_{k}=e_{2}^{U} \\
A_{k}^{L} x_{k}+B_{k}^{L} y_{k} \leq e_{1 k}^{L} \\
C_{k}^{L} x_{k}+D_{k}^{L} y_{k}=e_{2 k}^{L} \\
p_{k} B_{k}^{U}+\mathrm{q}_{k} D_{k}^{U}+r_{k} B_{k}^{L}-t_{k}=-\theta_{k}^{L} \\
p_{k}\left(e_{1}^{U}-A^{U} x-\sum_{k=1}^{K} B_{k}^{U} y_{k}\right)+r_{k}\left(e_{1 k}^{L}-A_{k}^{L} x_{k}-B_{k}^{L} y_{k}\right)+t_{k} y_{k}=0 \\
x_{k} \geq 0, y_{k} \geq 0, p_{k} \geq 0, q_{k} \geq 0, r_{k} \geq 0, t_{k} \geq 0 \\
k=1,2, \ldots, K
\end{array}\right.
\end{aligned}
$$

\section{Case study: an unattended convenience store}

\subsection{Data description}

Auchan (AU) is the second largest retailer in France and one of the top ten retailers in the world. As a leading retailer, the company began to establish unattended convenience stores in November 2017 and gradually formed a model of a single control center with multiple retail boxes. This study will evaluate the benefit efficiency of seven AU unattended convenience store systems in Chengdu, China. The data were provided by the person in charge of the AU unattended convenience stores in Chengdu.

The benefit efficiency of each system is determined using the following nine variables. At the upper level, the control center's personnel cost $X_{L}^{S}$ and logistics cost $X_{L}^{D}$ as the control center's input to produce the intermediate measures, including desirable frequency of delivery $Y_{L F k}^{G}$ and undesirable shortage rate output $U_{L F k}^{B}$, which link the upper level and lower level. At the lower level, the retail boxes' personnel cost $X_{F k}^{S}$, rent $X 1_{F k}^{D}$, promotion rate $X 2_{F k}^{D}$ and above intermediate measures as the retail boxes' input to produce turnover $Y 1_{F k}$ and the number of customers $Y 2_{F k}$ as final output. The raw data of these variables is shown as Table 1 (CC: control center; RB: retail box). 
Table 1. Raw data

\begin{tabular}{|c|c|c|c|c|c|c|c|c|c|}
\hline \multirow[t]{2}{*}{ DMU } & \multicolumn{2}{|c|}{ CC's input } & \multicolumn{2}{|c|}{$\begin{array}{c}\text { Intermediate } \\
\text { measures }\end{array}$} & \multicolumn{3}{|c|}{ RB's input } & \multicolumn{2}{|c|}{ RB's output } \\
\hline & $\mathbf{X}_{\mathrm{L}}^{S}$ & $\mathrm{X}_{\mathrm{L}}^{\mathrm{D}}$ & $V_{\mathrm{LFk}}^{\mathrm{G}}$ & $\mathrm{U}_{\mathrm{LFk}}^{\mathrm{B}}$ & $\mathrm{X}_{\mathrm{Fk}}^{\mathrm{S}}$ & $\mathrm{X} 1_{\mathrm{Fk}}^{\mathrm{D}}$ & $\mathrm{X} 2_{\mathrm{Fk}}^{\mathrm{D}}$ & $\mathrm{Y1}_{\mathrm{Fk}}$ & $\mathrm{Y} 2_{\mathrm{Fk}}$ \\
\hline \multirow[t]{5}{*}{1} & 4650 & 3000 & 15 & 5.80 & 1850 & 0 & 7.60 & 1228 & 653 \\
\hline & & & 15 & 4.20 & 1850 & 0 & 7.40 & 3298 & 1471 \\
\hline & & & 8 & 11.1 & 1850 & 1500 & 10.0 & 810 & 287 \\
\hline & & & 8 & 13.5 & 1850 & 1000 & 7.80 & 913 & 354 \\
\hline & & & 8 & 9.80 & 1850 & 1000 & 6.90 & 1146 & 404 \\
\hline \multirow[t]{5}{*}{2} & 5120 & 2500 & 8 & 11.1 & 2100 & 1000 & 8.60 & 1503 & 490 \\
\hline & & & 8 & 9.60 & 2100 & 1000 & 8.10 & 1762 & 727 \\
\hline & & & 8 & 10.1 & 2100 & 800 & 7.50 & 649 & 240 \\
\hline & & & 8 & 11.4 & 2100 & 0 & 8.00 & 2065 & 612 \\
\hline & & & 8 & 9.70 & 2100 & 1000 & 7.90 & 856 & 283 \\
\hline \multirow[t]{5}{*}{3} & 4700 & 2500 & 8 & 10.2 & 1950 & 1500 & 7.50 & 549 & 183 \\
\hline & & & 15 & 6.10 & 1950 & 0 & 8.10 & 3598 & 1870 \\
\hline & & & 15 & 5.30 & 1950 & 0 & 8.30 & 4576 & 1871 \\
\hline & & & 8 & 10.3 & 1950 & 1000 & 10.4 & 756 & 199 \\
\hline & & & 8 & 9.80 & 1950 & 1000 & 11.2 & 1555 & 444 \\
\hline \multirow[t]{5}{*}{4} & 4750 & 3000 & 8 & 11.1 & 1910 & 1000 & 10.7 & 1319 & 370 \\
\hline & & & 8 & 9.40 & 1910 & 1000 & 9.90 & 1549 & 446 \\
\hline & & & 8 & 10.1 & 1910 & 1500 & 10.4 & 1541 & 527 \\
\hline & & & 15 & 4.20 & 1910 & 0 & 9.80 & 3448 & 1149 \\
\hline & & & 15 & 5.60 & 1910 & 0 & 9.40 & 2806 & 1045 \\
\hline \multirow[t]{5}{*}{5} & 5300 & 2600 & 8 & 10.7 & 2050 & 1200 & 10.0 & 1407 & 409 \\
\hline & & & 8 & 9.50 & 2050 & 1200 & 9.60 & 2065 & 557 \\
\hline & & & 8 & 11.1 & 2050 & 1000 & 12.4 & 1616 & 447 \\
\hline & & & 8 & 10.8 & 2050 & 1000 & 11.1 & 2471 & 762 \\
\hline & & & 8 & 9.60 & 2050 & 1200 & 12.1 & 2328 & 719 \\
\hline \multirow[t]{5}{*}{6} & 4960 & 2900 & 8 & 10.4 & 2100 & 1200 & 9.80 & 2460 & 572 \\
\hline & & & 8 & 9.70 & 2100 & 1200 & 12.1 & 1699 & 458 \\
\hline & & & 8 & 10.6 & 2100 & 1000 & 11.4 & 2394 & 732 \\
\hline & & & 8 & 9.60 & 2100 & 1200 & 12.6 & 384 & 109 \\
\hline & & & 8 & 9.70 & 2100 & 1000 & 14.2 & 1536 & 434 \\
\hline \multirow[t]{5}{*}{7} & 4000 & 2000 & 8 & 10.8 & 2000 & 1000 & 8.10 & 943 & 490 \\
\hline & & & 15 & 5.60 & 2000 & 1000 & 8.10 & 3196 & 1537 \\
\hline & & & 15 & 6.40 & 2000 & 1000 & 8.80 & 2827 & 1165 \\
\hline & & & 8 & 10.6 & 2000 & 1000 & 9.20 & 1495 & 645 \\
\hline & & & 15 & 6.00 & 2000 & 1000 & 8.90 & 3652 & 1610 \\
\hline
\end{tabular}

\subsection{Results and discussion}

After processing the data of the unattended convenience stores in the Chengdu area using the model, the BEs of the lower subsystem, the upper subsystem and the entire system can be obtained.

As seen from Table 2, all the unattended convenience store systems of the brand in Chengdu are benefitinefficient. The main reason is that the upper and lower subsystems of each DMU are benefit-inefficient at the same time. Although the upper subsystem of DMU7 is benefit-efficient, the lower subsystem of DMU7 is benefitinefficient, and thus DMU7 is not efficient. However, overall, DMU7's system benefit efficiency is better than that of other DMUs.
Table 2. BEs of MFBLP-DEAwUIM.

\begin{tabular}{cccc}
\hline DMU & \multicolumn{3}{c}{ Benefit Efficiency (BE) } \\
\cline { 2 - 4 } & $\begin{array}{c}\text { Entire } \\
\text { system }\end{array}$ & $\begin{array}{c}\text { Lower } \\
\text { level }\end{array}$ & $\begin{array}{c}\text { Upper } \\
\text { level }\end{array}$ \\
\hline 1 & 0.85 & 0.43 & 0.95 \\
2 & 0.60 & 0.37 & 0.66 \\
3 & 0.84 & 0.63 & 0.89 \\
4 & 0.83 & 0.57 & 0.90 \\
5 & 0.64 & 0.51 & 0.67 \\
6 & 0.62 & 0.43 & 0.67 \\
7 & 0.94 & 0.71 & 1.00 \\
\hline
\end{tabular}

Moreover, compared with the upper-level, lower-level and overall BEs of DMUs, as shown in Figure 2, it can be concluded that the entire system BE of DMU is always between the upper-level BE and lower-level BE. 


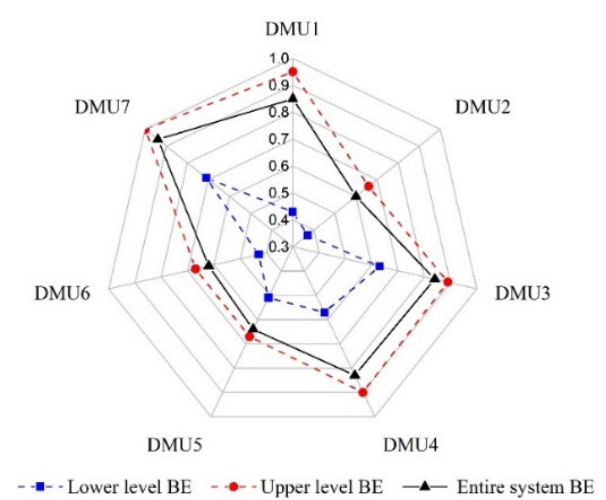

Figure 2. The upper level, lower level and Entire system BEs based on MFBLP-DEAwUIM

In addition, the BEs of each retail box in the lower subsystem can also be obtained using proposed model. From the BEs of the five followers listed in Table 3, we can see that the followers of each DMU are not benefitefficient at the same time, which results in the benefit inefficiency of all the lower subsystems of DMU. Although Followers 2 and 3 of DMU3 are benefit-efficient, the lower subsystems of DMU 3 are benefit-inefficient because other followers of the same subsystem are ineffective. The same is true for DMU4, DMU6 and DMU7.

Table 3. Lower-level BEs of MFBLP-DEAwUIM

\begin{tabular}{ccccccc}
\hline $\begin{array}{c}\text { DM } \\
\mathbf{U}\end{array}$ & \multicolumn{5}{c}{ Benefit Efficiency (BE) } \\
\cline { 2 - 7 } & $\begin{array}{c}\text { Lowe } \\
\boldsymbol{r} \\
\text { level }\end{array}$ & $\begin{array}{c}\text { Followe } \\
\boldsymbol{r} \mathbf{~}\end{array}$ & $\begin{array}{c}\text { Followe } \\
\boldsymbol{r} \mathbf{~}\end{array}$ & $\begin{array}{c}\text { Followe } \\
\boldsymbol{r} \mathbf{3}\end{array}$ & $\begin{array}{c}\text { Followe } \\
\boldsymbol{r} \mathbf{4}\end{array}$ & $\begin{array}{c}\text { Follower } \\
\boldsymbol{5}\end{array}$ \\
\hline 1 & 0.43 & 0.60 & 0.87 & 0.17 & 0.28 & 0.30 \\
2 & 0.37 & 0.64 & 0.46 & 0.14 & 0.58 & 0.22 \\
3 & 0.63 & 0.24 & 1.00 & 1.00 & 0.21 & 0.38 \\
4 & 0.57 & 0.54 & 0.36 & 0.32 & 1.00 & 0.73 \\
5 & 0.51 & 0.58 & 0.48 & 0.32 & 0.70 & 0.58 \\
6 & 0.43 & 1.00 & 0.39 & 0.48 & 0.11 & 0.37 \\
7 & 0.71 & 0.46 & 0.87 & 0.62 & 0.47 & 1.00 \\
\hline
\end{tabular}

Figure 3 shows the relationship between the lowerlevel BE of each DMU and the BEs of all its followers. We can see that the lower subsystem BE is always in the range of the maximum and minimum $\mathrm{BE}$ of the followers.

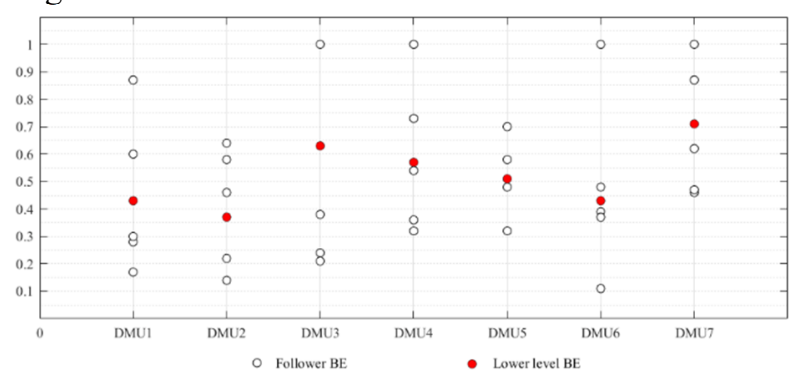

Figure 3. BEs of followers and the lower level subsystem based on MFBLP-DEAwUIM

Figure 4 is a scatter diagram based on the expected output data of the unattended convenience store system. From the plot, we can see that Follower 3 of the lower subsystem of DMU3 performs the best, and Follower 4 of the lower subsystem of DMU6 performs the worst. However, this does not accurately reflect the operational efficiency of all retail boxes. For example, according to the model we provide, Follower 5 of DMU7 can also be benefit-efficient. Moreover, the BE of the entire DMU is unknown. Therefore, simple statistical analysis based on some indicators cannot accurately reveal the efficiency of unmanned convenience store systems. On the other hand, this simply proves the validity of our model to some extent.

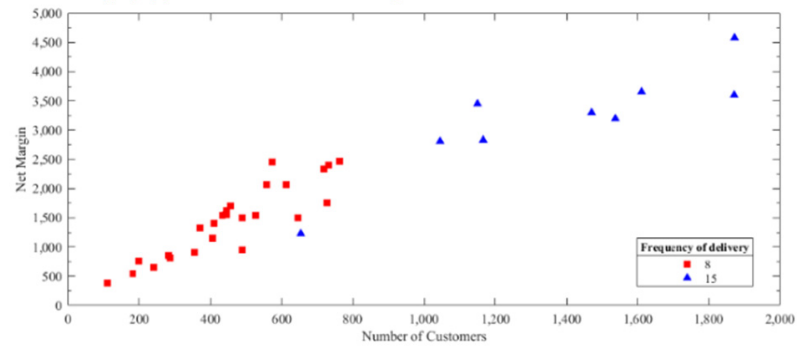

Figure 4: Frequency of delivery, turnover and number of customers

Furthermore, although the traditional "black box" CCR-DEA model can evaluate the overall efficiency of DMU, it only considers the initial input and final output of the system and ignores the internal operation of the unattended convenience store system. This model does not accurately identify which participants in the system should be responsible for the benefit inefficiency of the overall system. Moreover, although existing BLP-DEA studies can partially demonstrate the structure of bi-level systems, they rarely consider the situation of the multi-follower or the existence of undesirable intermediate measures, which makes it impossible to accurately and reasonably evaluate the efficiency of all scenarios similar to our research case.

In summary, the model we provide can clearly reveal the internal bi-level structure of the unattended convenience store system and, in particular, reflect the benefit efficiency of each retail box, upper and lower subsystems and the overall system and discover the reasons for its benefit inefficiency, which, in turn, can enable managers to focus on improving a specific participant in order to improve the $\mathrm{BE}$ of unattended convenience stores.

Insights for managers from the case study of unattended convenience stores include:

(1) The BE of the unattended convenience store is always between the control center subsystem and the retail box subsystem BE.

(2) The BE of the lower subsystem of unattended convenience stores always falls between the maximum BE and the minimum $\mathrm{BE}$ of all retail boxes in the system.

(3) The overall BE of DMU7 is relatively high. Although the lower subsystems of DMU7 have relatively high BE, their Followers 1 to 4 show potential room for improvement. Overall, the upper and lower BE of DMU2 are the smallest and must be focused on. In this case, study, all possible improvements can be considered in terms of personnel costs, logistics costs, rents, promotional proportions and how to distribute them more reasonably.

\section{Conclusions}

Evaluating the potential benefits of unattended convenience stores and other organizations similar to the operation structure of unattended convenience stores is an important decision-making problem faced by relevant managers and involves many input and output factors that play a role at the same time. 
In this paper, we study the leader-follower game structure from the perspective of the operation of an unattended convenience store system. The control center that undertakes the overall scheduling and distribution business is regarded as the leader, while the retail network comprising retail boxes is regarded as the follower. We define the concept of benefit efficiency under this structure and propose a specific BLP-DEA model (MFBLP-DEAwUIM) for this operation structure. The model uses weak disposability technology to deal with the undesirable intermediate measures, which has not been taken into account in previous studies of multi-follower DEA. In addition, we have developed a method to solve the model we proposed. Within this framework, we have proven the BE of a system with such a leader-follower structure is between that of the leader and follower. It can also be deduced that when both leader and follower are benefit-efficient, the system is also benefit-efficient. Through the analysis of 7 unattended convenience stores and 35 retail boxes in Chengdu, the results show that the control center subsystem, follower subsystem and retail box have significant potential benefits. The results of the case study also provide practical suggestions for managers of unattended convenience stores, which play an important role in identifying inefficient game players and improving them. Simultaneously, the proposed model can also play an important role in evaluating the potential benefits of the same type of organization.

Our model also has obvious limitations. Although the model can clearly and accurately explain the internal operation structure of the research object in the case, compared with input and output variables, the number of DMUs is very limited, which may weaken the recognition ability of the model [14]. Furthermore, for different DMUs, the allocation of efficiency weights to the desirable outputs by decision-makers may be inconsistent. In future studies, it may be worthwhile to use panel data to evaluate the benefit efficiency of the research subjects. The output variables of this paper primarily consider the economic dimension, but the transformation effect of unattended convenience stores on social development is more obvious, and thus, its social value can also be considered in future research.

\section{Acknowledgment}

First of all, I would like to thank my tutor for his patient guidance and detailed suggestions on my thesis, which has guided the direction of my thesis writing process. Secondly, I am also very grateful to my friends for their encouragement and help in the process of writing the paper. Through the communication with them, I have obtained many useful ideas and references, which makes me feel inspired in the process of writing the paper. Finally, I would like to thank the authors in the references. Reading their articles broadened my scientific vision, which gave me more inspiration to write papers.

\section{References}

1. A. Charnes, W. W. Cooper, and E. Rhodes, "Measuring efficiency of decision-making units," European Journal of Operational Research, vol. 2, no. 6, pp. 429-444, 1978.

2. R. D. Banker, A. Charnes, and W. W. Cooper, "Some models for estimating technical and scale inefficiencies in data envelopment analysis," (in English), Management Science, Article vol. 30, no. 9, pp. 1078-1092, 1984.

3. R. Fare and G. Whittaker, "An intermediate input model of dairy production using complex survey data," Journal of Agricultural Economics, vol. 46, no. 2, pp. 201-213, May 1995.

4. R. Fare and S. Grosskopf, "Productivity and intermediate products: A frontier approach," Economics Letters, vol. 50, no. 1, pp. 65-70, Jan 1996.

5. Y. Chen, J. A. Du, H. D. Sherman, and J. Zhu, "DEA model with shared resources and efficiency decomposition," European Journal of Operational Research, vol. 207, no. 1, pp. 339-349, Nov 2010.

6. L. Liang, F. Yang, W. D. Cook, and J. Zhu, "DEA models for supply chain efficiency evaluation," Annals of Operations Research, vol. 145, pp. 35-49, 2006.

7. D. D. Wu, "BiLevel programming data envelopment analysis with constrained resource," European Journal of Operational Research, vol. 207, no. 2, pp. 856-864, Dec 2010.

8. D. D. Wu, C. C. Luo, H. F. Wang, and J. R. Birge, "Bilevel programing merger evaluation and application to banking operations," Production and Operations Management, vol. 25, no. 3, pp. 498-515, Mar 2016.

9. J. Lu, C. G. Shi, and G. Q. Zhang, "On bilevel multifollower decision making: General framework and solutions," Information Sciences, vol. 176, no. 11, pp. 1607-1627, Jun 2006.

10. X. Y. Zhou, R. Luo, Y. Tu, B. Lev, and W. Pedrycz, "Data envelopment analysis for bi-level systems with multiple followers," Omega-International Journal of Management Science, vol. 77, pp. 180-188, Jun 2018.

11. O. Benayed and C. E. Blair, "Computational difficulties of bilevel linear-programming," Operations Research, vol. 38, no. 3, pp. 556-560, May-Jun 1990.

12. E. Roghanian, M. B. Aryanezhad, and S. J. Sadjadi, "Integrating goal programming, Kuhn-Tucker conditions, and penalty function approaches to solve linear bi-level programming problems," Applied Mathematics and Computation, vol. 195, no. 2, pp. 585-590, Feb 2008.

13. C. G. Shi, J. Lu, and G. Q. Zhang, "An extended Kuhn-Tucker approach for linear bilevel programming," Applied Mathematics and Computation, vol. 162, no. 1, pp. 51-63, Mar 2005.

14. D. D. Wu and J. R. Birge, "Serial chain merger evaluation model and application to mortgage 
banking," Decision Sciences, vol. 43, no. 1, pp. 5-35,

Feb 201 